\title{
Computational Analysis of Axial Compressor Stages Characteristics
}

\author{
A.I. BOROVKOV, YU.B. GALERKIN, YU.A. POPOV, A.F. REKSTIN, O.A. SOLOVYOVA, A.A. DROZDOV, L.N. \\ MARENINA, CHERNIKOV V.A. \\ Leading Research Center "Digital Design and Modeling (Smart Design)" \\ Peter the Great St.Petersburg Polytechnic University \\ 195251, St.Petersburg, Polytechnicheskaya, 29 \\ RUSSIA
}

\begin{abstract}
Published data on flat grid purges allow to evaluate the efficiency of blade devices with different kinematic scheme of stages and design parameters. The corresponding computer program ODOS-GP04 and examples of numerical analysis of the efficiency of flat grids and spatial blade devices of axial stages with different combinations of design parameters was provided. Further development of the ODOS-GP04 program is described below, which makes it possible to perform an estimated calculation of the gas-dynamic characteristics of the stages designed using this program. Analysis of the calculated characteristics of a number of typical stages allows to draw a conclusion about the influence of the main design parameters. This article is primarily addressed to people who are developing their knowledge of the gas dynamics of axial compressors. Some results of calculations do not correspond to the published experimental data. The authors expect that this may attract the attention of specialists working in the field of gas dynamics of axial compressors.
\end{abstract}

Key-Words: - Mathematical simulation, axial compressor, impeller, guiding device, efficiency, loss coefficient, theoretical head, velocity triangle, bushing ratio

Received: January 29, 2020. Revised: July 17, 2020. Accepted: August 16, 2020. Published: August 25, 2020.

\section{Legend}

$B$ - blade chord

$c$ - absolute velocity (flow rate in fixed coordinate

system);

$c_{a}-$ lift coefficient

$c_{w}$ - drag force coefficient

$F_{D I m}=1-w_{2} / w_{3}$ max. - diffusion airfoil factor of Im bucket

$F_{D G D}=1-c_{2} / c_{3} \max$ - diffusion airfoil factor of GD bucket

$h_{T}$ - ducted head

$h_{w}$ - loss of head;

$i_{1}=\beta_{b l l}-\beta_{1}, i_{2}=\alpha_{b l 2}-\alpha_{2}-$ incidence angle at the inlet

to the impeller, guiding device

$l / B$ - blades elongation (ratio of the blade height to

the chord at the design diameter)

$p$ - pressure

$t$ - grid spacing (distance between blades)

$T$ - temperature

$w$ - relative velocity (flow rate in a rotating coordinate system);

$u$ - circumferential velocity;

$\bar{V}$ - volume flow rate

$\alpha$ - angle between the absolute velocity and the

circumferential direction;

$\beta$ - angle between the relative velocity and the

circumferential direction; $\alpha_{b l}$ - guiding device vane angle

$\beta_{b l}$ - impeller blade angle

$\zeta$ - loss of head coefficient in grid

$\varepsilon_{1}=\beta_{2}-\beta_{1}, \varepsilon_{2}=\alpha_{2}-\alpha_{1}-$ flow turning angle by

impeller, guiding device blades

$\varphi=c_{z} / u=w_{z} / u$ - flow coefficient

$\phi_{h}=\frac{\bar{V}}{\pi r_{h}^{2}\left(1-\left(r_{h u b} / r_{h}\right)^{2}\right) u_{h}}$ - flow coefficient by outer diameter

$\Phi=\frac{\bar{m}}{\rho_{1}^{*} \pi r_{h}^{2} u_{h}}=\frac{\rho_{1} \bar{V}_{1}}{\rho_{1}^{*} \pi r_{h}^{2} u_{h}}$ - conditional flow coefficient

$\bar{\Phi}=\Phi \cdot \lambda_{u h^{-}}$dimensionless flow rate

$\lambda_{u}=\frac{u}{\sqrt{\frac{2 k}{k+1} R T_{1}^{*}}}$ - conditional velocity coefficient

$p=p_{3} / p_{1}$ - pressure ratio developed by stage

$\rho$ - gas density

$\eta=1-\frac{h_{w}}{h_{T}}$ - polytropic efficiency

$\psi_{T}=\left(w_{u l}-w_{u 2}\right) / u$ - loading factor

$\psi_{T h}$ - loading factor at the outer radius of impeller

$\Omega=1-\left(w_{u l}+w_{u 2}\right) / 2 u$ - "theoretical" degree of reaction
Abbreviations
GD - guiding device
Im - impeller

\section{Subindexes}


$1,2,3$ - flow parameters at inlet and outlet of the impeller and guiding device bucket;

GD - guiding device grid;

$\mathrm{d}$ - related to design mode (by consumption)

des - parameters on the design radius

ImBS - impeller bucket system;

$\mathrm{u}$ - speed projection on circumferential direction

$\mathrm{z}$ - speed projection on axial direction

\section{Superscript}

* - refers to total parameters.

\section{Introduction}

Published data on flat grid purges allow to evaluate the efficiency of blade devices with different kinematic scheme of stages and design parameters. The corresponding computer program ODOS-GP04 and examples of numerical analysis of the efficiency of flat grids and spatial blade devices of axial stages with different combinations of design parameters are presented in the articles [1], [2]. Further development of the ODOS-GP04 program is described below, which makes it possible to perform an estimated calculation of the gas-dynamic characteristics of the stages designed using this program. Analysis of the calculated characteristics of a number of typical stages allows to draw a conclusion about the influence of the main design parameters. Like previously published works [1], [2], this article is primarily addressed to people who are developing their knowledge of the gas dynamics of axial compressors. Some results of calculations do not correspond to the published experimental data. The authors expect that this may attract the attention of specialists working in the field of gas dynamics of axial compressors.

\section{Literature review}

In the Russian literature, the principles of profiling blade devices of axial compressors based on flat grids testing were described in [3], and in more detail in [4]. The monograph [5] presents the results of extensive research and design methods applied to axial fans. Recommendations for the design of industrial axial compressors are contained in the technical materials [6] and others. A number of specific design issues have been solved by domestic researchers and described in a number of publications, for example, in [7]. The works [8], [9] present the development of a software package for the optimization of size and form of axial compressors. The developed mathematical model takes into account various components of head losses in axial compressors and allows optimizing their dimensions, impeller and guiding device blades form, and provides predicted gas-dynamic characteristics of the compressor. The work [10] presents a mathematical model for calculating the characteristics of multistage axial compressors. It is an extended one-dimensional model for calculating the flow parameters on the center current line along the blade height, which the authors themselves call 1.5D. The authors present the results of verification of their model for two axial compressors. The work [11] presents the concept of the through flow method. In recent decades, the through flow method has become one of the most important tools for designers - a compromise between the accuracy of the flow field representation and the corresponding computational cost. The work [12] presents a program for optimizing the impeller blades of axial compressor stages based on a proprietary CFD program. The authors created an automatic algorithm for computational grid rebuilding and an optimizer that allows varying the geometry of the blade surfaces on the current lines to achieve a given pressure distribution on the blades. The work [13] presents a program for preliminary evaluation of the layout and form of axial turbomachines of transport GTE. The program is designed to analyze a variety of options for the flow part and select the optimal number of compressor stages, the meridional form of the flow path, etc. The authors note that the use of pre-design programs is an integral step in the creation of new GTE. The work [14] presents an improved model for transonic axial compressors calculation and design based on two-dimensional calculations. The model was verified based on experimental studies of a transonic axial stage and a three-stage $\mathrm{P} \& \mathrm{~W}$ 3S1 compressor. The work [15] presents a program for preliminary design of an axial multistage compressor. The calculation and optimization in it is carried out for the center current line along the height of the compressor blades. The underlying mathematical model allows also to design supersonic compressors. However, the loss calculation is performed only for direct shock waves and the calculation algorithm does not take into account changes in flow parameters after shock wave. This slightly reduces the functionality of this program. In works [16], [17] the code of the center line of an axial compressor operating in non-calculating modes is described for specialized engineering software that is developed for the analysis of the entire gas turbine engine. A generalized methodology for losses simulation on the midline of the axial compressor has been developed. A number of researchers only use CFD calculations to optimize the form of axial compressor stages. An example of such work is the 
publication [18]. In work [19], a three-stage axial compressor with an inlet guiding device operating with a transonic flow pattern is calculated. The influence of accounting for leaks through labyrinth seals on compressor characteristics is studied. Computer programs based on the flow organization principle and mathematical models well in relation to compressors with subsonic gas movement [20].

\section{Research results}

\subsection{Main provisions and design capabilities of the ODOS-GP04 program}

At the stage of dimension selection and calculation of profile losses in a given mode, the program operates with so-called homogeneous stages - at the calculated radius, the velocity flow components are the same at the inlet to the Im and GD $\left(c_{z 1}=c_{z 2}\right)$, the incompressible gas moves along cylindrical surfaces.

At the first stage, the program sets the main parameters of flat grids of blade devices at the calculated radius, based on the user-selected parameters:

- degree of reaction,

- flow coefficient,

- Im blades pitch ratio,

- diffusion airfoil factor [21] of Im and GD bucket.

Using these parameters, the program calculates Im loading factor and GD blades pitch ratio (relative pitch of GD blades can be set, then the program calculates the corresponding GD diffusion airfoil factor). The profile loss coefficient is calculated by the empirical dependence from [21] with the recommended optimal value $\mathrm{FD}=0.45$. For other values, the authors in [1] proposed close approximation of the experimental data.

At the second stage, the user can set the hub-tip ratio of the stage and choose one or another spatial flow law based on the ratio $\boldsymbol{c}_{\boldsymbol{u}} \boldsymbol{r}^{\boldsymbol{m}}=$ const. Here $\mathrm{m}$ is a user-selectable stage exponent. The value of this indicator within the limits $\mathbf{\pm} \mathbf{1}$ determines the nature of changes in the circumferential and axial components of the velocities along the height of the blades [4]. The calculation of velocity triangles on a number of current surfaces, the values of the diffusion airfoil factor and profile losses makes it possible to qualitatively and quantitatively analyze different versions of stages.

The presented final part of the ODOS-GP04 program allows to make an estimated calculation of stages characteristics, the parameters and dimensions of which are set at the specified performance mode at the previous stages. When forming a system of equations, simplified representations of pressure losses in non-calculated modes are used. Therefore, the results of calculations should be considered as a demonstration of trends, and not as reliable quantitative data.

\subsection{The dimensionless representation of the gas-} dynamic characteristics

When presented graphically, the ODOS-GP04 program allows the user to apply three different arguments to demonstrate functional dependencies stage characteristics and flow parameters in the Im and GD. Since all the results are presented in a tabular format, the user can independently build any necessary graphs.

\subsection{Characteristics of model stages}

The conditional flow coefficient $\Phi$ determines the mass productivity of the stage, so during calculation the change in density at the inlet to Im depending on the velocity coefficient should be taken into account:

$$
\rho_{1} / \rho_{1}^{*}=\left[1-\frac{k-1}{k+1}\left(\frac{\lambda_{u h}}{r_{\mathrm{i}} / r_{\text {des }}}\right)^{2}\left(c_{1} / u\right)^{2}\right]^{1 / k}
$$

It is convenient to use the coefficient $\Phi$ for constructing the characteristics of model stages, since it allows to determine the mass flow rate of a real compressor stage without unnecessary calculations. The conditional velocity coefficient $\lambda_{u h}$ at the impeller shroud is used as a parameter when presenting characteristics. The functions are the polytropic efficiency, the polytropic head coefficient by the outer diameter, and the power factor:

$$
\eta, \psi_{h}, \bar{N}=f\left(\Phi, \lambda_{u h}\right)
$$

Where: $\bar{N}=\Phi \cdot \psi_{T h}$.

\subsection{Parameters in function of the dimensionless flow rate}

When constructing a family of dimensional characteristics of the compressor (stage), with rotor speed increase, the characteristics shift towards flow rate and pressure ratio increase. When the inlet flow parameters are set, increase in the rotor velocity causes a proportional increase in the velocity coefficient $\lambda_{u h}$. When presenting the characteristics in a dimensionless form, the role of the rotor velocity is demonstrated by introducing the product $\bar{\Phi}=\Phi$. $\lambda_{u h}$ as argument of dimensionless flow functions:

$$
\eta, \psi_{h}, \bar{N}=f\left(\bar{\Phi}, \lambda_{u h}\right)
$$

\subsection{Parameters in function of the flow coefficient}

This coefficient is uniquely related to the volume flow rate, and its use as an argument for gas-dynamic 
parameters functions is appropriate when the flow kinematics in the stage at different flow rates is of interest. The characteristics of the stage as a function from the flow coefficient at the calculated radius are:

$$
\eta, \psi_{\text {Tdes }}, \Pi=f\left(\phi_{\text {des }}, \lambda_{\text {uh }}\right)
$$

Let us recall, that the subscript "des" indicates that the parameter belongs to the average calculated radius. In addition, as a function from flow coefficient, the efficiency losses in the Im and GD and the drag force coefficients - profile and on limiting surfaces, can be represented.

\subsection{Efficiency of the stage at designed output mode}

When calculating this efficiency in part 2 of the ODOS-GP04 program, only profile losses are calculated, and the possible negative impact of high velocities (gas compressibility) on head losses is not taken into account. This must be done when calculating characteristics. To calculate the loss of efficiency in the Im and GD, knowledge of the loss coefficient, the loading factor, and the dimensionless kinetic energy at the inlet is required. For example, for the Im:

$$
\Delta \eta_{I m}=\frac{\zeta_{I m}}{\psi_{\mathrm{T}}} \frac{\left(w_{1} / u\right)^{2}}{2}
$$

3.7 Calculation of efficiency taking into account losses on the surfaces of the housing and rotor (limiting surfaces).

The monograph of N. Cumpsty [22] indicates that the approach recommended by A. Howell in the 40s of the last century [23] to the estimated calculation of these losses is still relevant. Losses are calculated as an addition to the profile drag force coefficient:

$$
c_{w}=c_{w p}+c_{w 0}+c_{w i}
$$

Here:

$\mathrm{c}_{\mathrm{w}}-$ drag force coefficient that takes into account all losses in the spatial grid,

$\mathrm{c}_{\mathrm{wp}}$ - profile drag force coefficient,

$\mathrm{c}_{\mathrm{w} 0}-$ drag coefficient due to friction on bounding surfaces,

$\mathrm{c}_{\mathrm{wi}}-$ drag coefficient due to secondary flows on bounding surfaces.

In fact, losses on bounding surfaces are determined by the behavior of the three-dimensional boundary layer. The division of losses into two components is conditional and corresponds to early ideas about the process. However, formula recommended for the assessment calculations give, according to N. Cumpsty, quite reasonable results:

$$
\begin{aligned}
c_{w 0}= & 0.02 \frac{t / B}{l / B}, \\
c_{w i} & =0.018 c_{a}^{2} .
\end{aligned}
$$

Here:

1/B - Im and GD blades elongation, which is selected by the designing engineer on the basis of strength and gas-dynamic considerations,

$\mathrm{ca}$ - lift force coefficient equal to the impeller and guiding device:

$$
\begin{array}{r}
c_{a I m}=2\left(\frac{t}{B}\right)_{I m} \frac{\psi_{T}}{w_{a v} / u} \\
c_{a G D}=2\left(\frac{t}{B}\right)_{G D} \frac{c_{u 2} / u-c_{u 1} / u}{c_{a v} / u}
\end{array}
$$

The definition of average vector velocities included in formulas $(7,7 \mathrm{a})$ is quite obvious and justified, for example, in [24].

The relationship of the drag force coefficient (or its components) with the loss coefficient follows from the equality of the lost power, defined as the product of the drag force on the average vector velocity, and as the product of the head loss on the mass flow rate. In the case of the impeller (for GD, the formula is similar):

$$
\zeta_{I m}=\frac{\left(c_{w p}+c_{w 0}+c_{w i}\right)_{I m}}{(t / B)_{I m}} \frac{\left(w_{a v} / u\right)^{3}}{\phi\left(w_{1} / u\right)^{2}}
$$

3.8 Accounting for the compressibility effect on the profile resistance (designed output mode)

Published data on purges of subsonic flat grids show that at the nominal mode, the profile drag force coefficient increases as the compressibility criterion increases slightly at first, and then faster. By analogy with modeling a similar phenomenon in the flow path of centrifugal stages [25], the authors introduced an empirical coefficient to account the compressibility effect on profile losses:

$$
\begin{aligned}
c_{w p p(\lambda)} & =K_{\lambda} \cdot c_{w p p}, \\
K_{\lambda} & =1+X_{i} \lambda_{\text {wmax }}^{X_{i}} .
\end{aligned}
$$

Here:

- $c_{w p}$ - profile drag force coefficient corresponding to the profile losses averaged by height of the blade in the design mode (calculated in part 2 of the ODOSGP04 program), 
$X_{i},-$ empirical coefficients whose values should ensure at least approximate compliance of the calculated characteristics with the test data.

Analysis of the centrifugal stages testing results showed that the most universal dependences are based on accounting of the maximum velocity on the profile [25]. This velocity occurs near the leading edge on the suction side of the blades. The ODOSGP04 program operates with the diffusion airfoil factor, which allows to determine this velocity:

$$
F_{D}=1-\frac{w_{2}}{w_{3 \max }}=1-\frac{w_{2} / w_{1}}{w_{3 \max } / w_{1}},
$$

from which

$$
w_{3 \max } / w_{1}=\frac{w_{2} / w_{1}}{1-F_{D}}
$$

In gas dynamics, one of the similarity criteria that equally determines the compressibility effect compressibility is used - the number $\mathrm{M}$, or the velocity coefficient $\lambda$. In this case, it is more convenient to use the velocity coefficient. This parameter is calculated from the total temperature, which is a constant value for the considered flow area. Taking into account that the total temperature in relative motion is greater than the total temperature at the stage inlet due to the kinetic energy of rotation $\mathrm{u} 2 / 2$

$$
\lambda_{w \max }=\lambda_{u h} \frac{r}{r_{h}}\left(w_{1} / u\right) \frac{\frac{w_{2} / w_{1}}{1-F_{D}}}{\sqrt{\left(1+\frac{k-1}{k+1}\left(\lambda_{u h} \frac{r}{r_{h}}\right)^{2}\right)}}
$$

The velocity coefficient in GD is calculated taking into account the increment of the total temperature in absolute motion (mechanical work input in the Im):

$$
\lambda u h \frac{r}{r_{12}} \frac{\frac{c_{1} / c_{2}}{1-F_{D G D}}}{\sqrt{1+2 \frac{k-1}{k+1} \psi_{T 0}\left(\lambda_{u h} \frac{r}{r_{h}}\right)^{2}}} .
$$

Formulas $(11,11 \mathrm{a})$ allow to calculate the value of the compressibility criterion on any of the current surfaces along the blade height. In the ODOS-GP04 program, the maximum velocity coefficient on the profile is calculated on the shroud, hub, and on the average calculated radius. The maximum of these values is used for calculations using the formula (9).

3.9 Stage efficiency at modes other than the calculated one. Losses on bounding surfaces.

According to formulas (6), the friction losses (coefficient $c_{w 0}$ ) are considered independent of the flow rate. Losses from secondary currents (coefficient $c_{w i}$ ) change with changes in the coefficients of lift force - formula $(7,7 \mathrm{a})$.

\subsection{Stage efficiency at modes other than the calculated one. Profile losses}

Dependence of the profile drag force coefficient on the incidence angle.

The results of purges given in the literature (for example, in [24]) show that in a certain range of small incidence angles, the profile drag force coefficient is almost constant, and then begins to increase sharply. As the Mach numbers (or velocity coefficient) increase, the range of minimum values of the profile drag force coefficient becomes narrower.

For a close approximation of the empirical relationships, the authors propose the following formula:

$$
\mathrm{c}_{\mathrm{wp}}\left(\mathrm{i}, \lambda_{\max }\right)=\mathrm{c}_{\mathrm{wpp}} *\left(\mathrm{~K}_{\lambda}+\mathrm{K}_{\mathrm{i}}\right)
$$

The incidence losses coefficient in the formula (12) is equal to zero within the range from $\mathbf{i}=\mathbf{0}$ to $\mathbf{i}=\mathbf{i}_{\text {пр }}$.

The value $\mathbf{i}_{\text {пр }}$ is suggested to be evaluated using the formula:

$$
\mathrm{i}_{\mathrm{ap}}=\mathrm{X}_{\mathrm{i}}-\mathrm{X}_{\mathrm{i}} \lambda_{\text {max }} \mathrm{X}_{\mathrm{i}}
$$

At incidence angles greater than ilim:

$$
\mathrm{K}_{\mathrm{i}}=\mathrm{X}_{\mathrm{i}}\left(\mathrm{i}_{1}-\mathrm{i}_{1 \mathrm{ap}}\right)^{\mathrm{X}_{\mathrm{i}}+\mathrm{X}_{\mathrm{i}} \lambda_{\max }}
$$

Figure 1 shows examples of dependencies $\mathrm{K}_{\mathrm{i} \lambda}=\mathrm{f}$ (i, $\lambda \mathrm{w}$ max) corresponding to one of the approximation options used in calculating the efficiency characteristics.

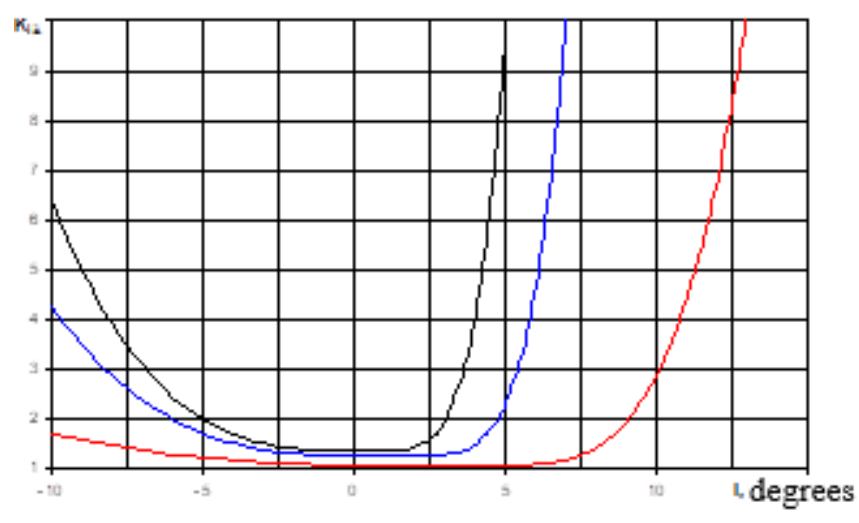

Fig. 1 The nature of the dependence $\mathrm{cwp}=\mathrm{f}(\mathrm{i}, \lambda \max )$, tried as one of the options for calculating the efficiency characteristic. $\lambda \max =0.4$ (red), 0.85 (blue), 1.0 (black).

It should be said that the characteristic of the efficiency $\boldsymbol{\eta}=\boldsymbol{f}(\boldsymbol{\Phi})$ shown in Fig. 1 in the form of a function $\boldsymbol{c}_{\boldsymbol{w} \boldsymbol{p}}=\boldsymbol{f}(\boldsymbol{i})$ also has a straight section in a 
certain flow range, which does not correspond to the test data of model stages. For this reason, incidence losses modeling using formulas (12) and (13) was not used in the final version of the program.

3.11 Dependence of the profile drag force coefficient on the incidence angle related to the nominal angle of rotation of the flow.

As it is known, the characteristics of plane grids can be generalized if they are represented as dependencies $\varepsilon / \varepsilon^{*}, c_{w p}=f\left(\left(i-i^{*}\right) / \varepsilon^{*}\right)-[22]$, [24]. Here, the superscript $*$ marks the so-called nominal mode - in our case, this is the designed output mode. At the same time, N. Cumpsty in [22] gives the results of calculating the characteristics of multi-stage compressors, using the dependence $c_{w p}=f\left(\left(i-i^{*}\right) / \varepsilon^{*}\right)$ in the form of a symmetric parabola - without a section in which the value $c_{w p}$ remains constant. However, the type of efficiency characteristic has more familiar character. The following type of approximation is used in the following calculations of characteristics, taking into account the above considerations and formulas (9), (12), (14):

$$
c_{w p}\left(i, \lambda w p p\left(1+X_{i} \lambda_{\max }^{X_{i}{ }^{X_{i}+X_{i} \lambda \max }}\right)_{\max }\right.
$$

The values of the empirical coefficients were selected by the authors in such a way that the calculated characteristics were similar in nature to the characteristics of one of the model stages published in [26].

\subsection{Dependence of the loading factor on the flow rate}

In a wide range of incidence angles, there is an almost linear dependence of the angle of flow rotation $\varepsilon=$ $\beta_{2}-\beta_{1}=f(i)-[24]$. This means that the flow outlet angles from the $\mathrm{Im}$ and GD $\beta 2$ and $\alpha 1$ practically do not differ from the values at the calculated mode. In this case, the velocity triangles imply:

$$
\psi_{T}=\left(1-\operatorname{ctg} \alpha_{1 \mathrm{p}}-\operatorname{ctg} \beta_{2 \mathrm{p}}\right) \phi .
$$

It follows from equation (15) that the loading factor depends linearly on the flow coefficient, increasing to one at zero flow.

\subsection{Change in the nature of the spatial flow at flow rates other than the calculated one}

The generally accepted method for constructing a spatial flow includes a condition $h_{T}(r)=\psi_{T} u^{2}=$ const ensuring that there is no mixing losses along the blades height. At the same time, in the design mode, the loading factors obey the law of change $\psi_{T}(r)=\psi_{T \mathrm{p}}\left(r_{\text {des }} / r\right)^{2}$.. That is, at the peripheral sections of the blades, the loading factors are significantly less than at the design radius, and at the hub - on the contrary. When the flow rate decreases in all cases, the values of the loading factors tend to unity - Fig. 2.

Let's assume that when the mode changes, the flow rates at all radii change in the same proportion. The nature of the change $\psi_{\mathrm{T}}=f\left(\bar{V} / \bar{V}_{\mathrm{p}}\right)$ in this case looks as shown in the figure above. If the flow rate decreases in the peripheral sections, the loading factor will increase to a greater extent than in the root sections, since in the design mode in the peripheral sections, the loading factor is smaller, and its dependence on the flow rate $\psi_{\mathrm{T} h}=f(\phi)$ goes steeper. As the flow rate increases, the pressure in the peripheral sections will decrease rapidly and may become negative. In both cases, the condition $\psi_{T}(r)=\psi_{T \mathrm{p}}\left(r_{\text {des }} / r\right)^{2}$. is violated.

The first consequence: In non-calculated flow modes, the mechanical energy of the gas after interaction with the impeller becomes unequal in the blades height. The flow that was supposed to be potential before the Im becomes a vortex after it. The inevitable process of equalizing mechanical energy in this case is carried out only due to the friction of gas layers with different energies. That is, in addition to the well-known losses due to shock flow around the blades in non-calculated modes, there are also inevitably mixing losses by the blades height.

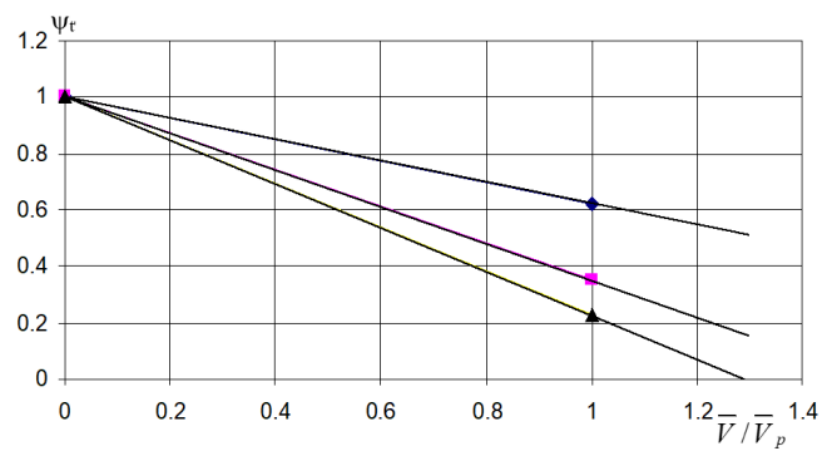

Fig. 2 Dependence of the loading factor on the relative flow rate for the outer, middle and hub radii.

Accepted: the hub-tip ratio is 0.6 , the calculated radius is the arithmetic mean, and the loading factor at the average radius is 0.35 .

The second consequence is a violation of the dependence of the flow rate on the blade height, which is typical for the design mode. Let's consider the well-known equation of radial equilibrium on a cylindrical surface of a current: 


$$
c_{z} \frac{\partial c_{z}}{\partial r}=\frac{1}{\rho} \frac{\partial p^{*}}{\partial r}-c_{u} \frac{\partial c_{u}}{\partial r}-\frac{c_{u}^{2}}{r}
$$

If a spatial flow with constant circulation was selected for the design mode: $\mathrm{h}_{\mathrm{T}}(\mathrm{r})=$ const, i.e. $\partial p^{*} / \partial r=0$ and $\mathrm{c}_{\mathrm{z}}(\mathrm{r})=$ const, $\mathrm{c}_{\mathrm{ur}}=$ const, then with the same decrease in the flow rate along the blade height, the pressure increases along the blade height, as shown above.

That is, in contrast to the design mode, the condition for the constancy of the total pressure over the blade height changes to $\partial p^{*} / \partial r>0$. In accordance with the equilibrium condition, instead of the condition $c_{z}(r)=$ const, the flow rate increases along the blade height. At flow rates more than the calculated one the opposite is true - hub velocity is greater than at the shroud. These conclusions are confirmed by measurements in real compressors at non-calculated modes [22].

\subsection{General procedure for calculating gas dynamic characteristics implemented in the ODOS-GP04 program}

When profiling blade devices by radius and calculating profile losses, the flow was analyzed as the sum of individual flows at different radii. Taking into account the diffusion airfoil factor reflects the velocity distribution on the blades. This means that the flow on each of the cylindrical surfaces is considered in a simplified, but still two-dimensional formulation. It seems logical and attractive to continue this approach even with flow rates that are not equal to the estimated one. For this purpose, the equilibrium equation and under condition $\partial p^{*} / \partial r \neq$ 0 can be solved numerically. However, for a program aimed at qualitative analysis, this approach seems unnecessarily complicated.

For this reason, the ODOS-GP04 program implements a simpler one-dimensional approach, which consists in the fact that the loss of efficiency from profile losses at the design mode, averaged by the blades height, refers to the average radius. Profile losses at non-calculated modes are calculated on the average radius and are considered to be averaged by the blades height.

Note. The set of characteristics obtained as a result of calculations for different $\lambda_{\text {uh }}$ is not a family of stage characteristics in the generally accepted sense. The family of characteristics is the characteristics of the one stage. When the compressibility changes in the stage, the ratio between the flow rate components changes, which follows from the continuity equation. In the ODOSGP04 program, the condition $c_{\mathrm{z} 1}=\mathrm{c}_{\mathrm{z} 2}=\mathrm{c}_{\mathrm{z} 3}$ is met in all cases at the calculated radius. That is, in fact, when calculating characteristics, different values of $\lambda u h$ correspond to different stages, which differ in the ratio of the flow area $f_{1} / f_{2} / f_{3}$.

\section{Discussion of the obtained results}

The ODOS-GP04 program provides very broad opportunities for computational analysis that cannot be exhausted in a separate publication. Some issues that, according to the authors, may attract the attention of specialists are discussed below.

\subsection{The subsonic stages of the limiting parameters}

For stages with different combinations of design parameters, the values $\lambda_{\text {uhcrit }}$ are determined for which the local values of the velocity coefficient do not exceed one on the blades of the Im and GD - in any of the sections by the blades height. Thus, for the stages the limit values of the head and flow rate are determined, at which the flow remains subsonic at the design mode. The objects of calculation studies:

- in all cases, the value of the diffusion airfoil factor $\mathrm{FD}=0.45$ is assumed for the Im and GD - on the average radius at the calculated output,

- the Im pitch ratio on the average radius is assumed to be equal to one,

- the blades chord is constant in their height,

- the relative elongation of the Im and GD blades is assumed to be equal to 2.5 ,

- stages with a reactivity degree of 0.5 and 1.0 are compared when constructing a spatial flow according to the law cur $=$ const, in the hub-tip ratio range $\mathrm{D}_{\mathrm{hub}} / \mathrm{D}_{\mathrm{h}}==0.5-0.9$ and flow coefficients at the calculated radius $\phi_{d e s}=0.35-0.80$,

- when calculating the pressure ratio, the adiabatic index is assumed to be $\mathrm{k}=1.4$.

Let's note that the calculations of the spatial flow did not take into account unfavorable factors in the form of increased values of the diffusion airfoil factor, excessive deceleration and rotation of the flow in individual sections along the blades height.

When considering the results for calculating the limit values $\lambda_{\text {uhcrit }}$ at which the local flow velocities do not exceed the sound velocity anywhere (Fig. 3a, $3 b$ ), we note that the maximum local velocity of the stages c $\Omega=1.0$ occurs at the shroud of the Im blades. At the same time at the stages with $\Omega=0.5$ the maximum local velocity occurs at the root of the guiding device blades. This difference (not taken into account in the simplest comparison of the kinematics of steps with different reactivity) determines the difference in the nature of dependencies $\lambda_{\text {uhcrit }}=$ $f\left(\phi_{d e s}, v\right)$ for stags with different reactivity. As expected, at the stage with $\Omega=0.5$, the maximum 
local velocity reaches the sound velocity at higher $\lambda_{\text {uhcrit }}$.

With an increase in the hub-tip ratio for stages with a reactivity of 1.0 , the critical velocity coefficient decreases rapidly due to an increase in the load of the shroud sections of the Im. For steps with a reactivity of 0.5 , the dependence $\lambda_{\text {uhcrit }}=f(v)$ is not so pronounced and has a different character at different $\phi_{\mathrm{p}}$.

As expected, regardless of the degree of reactivity, the loading factors on the outer diameter increase with the growth of the hub-tip ratio and the calculated flow coefficient. At the same time, stages with higher reactivity have higher loading factor.

However, this does not compensate for the low level of $\lambda_{\text {uhcrit }}$, so the limiting pressure ratio of subsonic stages is noticeably lower than that of stages with a reactivity of 0.5 - Fig. $4 \mathrm{a}, 4 \mathrm{~b}$. Let's note that for stages with a reactivity of 1.0 , the highest pressure ratio is achieved with a calculated flow coefficient of 0.5 . For stages with a reactivity of 0.5 , the lower the calculated flow coefficient, the higher the limit pressure ratio. In this case, the role of $\lambda_{\text {uhcrit }}$ prevails. It is also necessary to keep in mind the role of the efficiency coefficient, which in all cases is noticeably lower for stages with higher reactivity (this is discussed below).
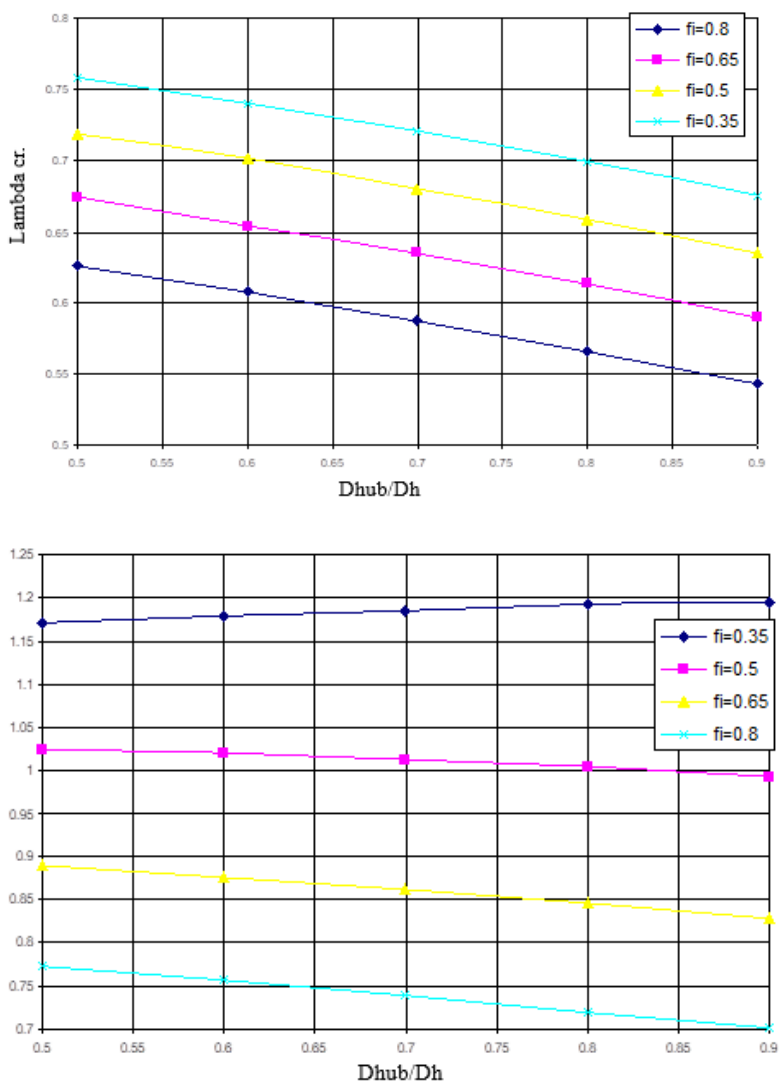

Fig. 3a, 3b. Influence of the hub-tip ratio and the calculated flow coefficient on the critical value of the velocity coefficient $\lambda_{\text {uhcrit }}$. At the top $\Omega=1.0$ - at the bottom $\Omega=0.5$.
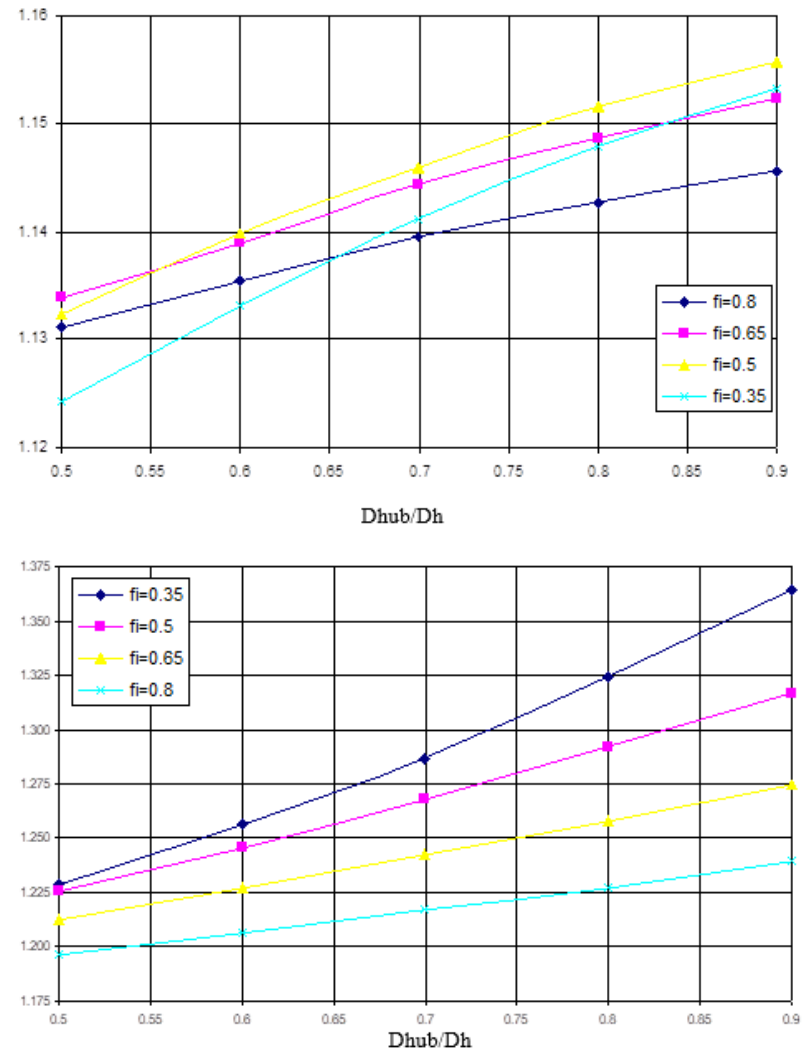

Fig. $4 a, 4 b$. Influence of the hub-tip ratio and the calculated flow coefficient on the pressure ratio $\mathrm{P}$ at the critical value of the velocity coefficient $\lambda_{\text {uhcrit }}$. At the top $\Omega=1.0$, at the bottom $\Omega=0.5$.

The maximum possible performance of subsonic stages is determined by the dimensionless flow rate

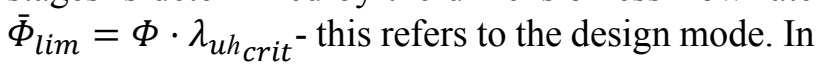
this case, local supersonic velocities will appear at modes with a flow rate greater than the calculated one.

The effect of the hub-tip ratio and the calculated flow coefficient on the maximum dimensionless flow rate is shown in Fig. 5a, 5b. For both types of stages, the value of the flow coefficient is the determining factor, not the critical velocity coefficient. Therefore, the dimensionless flow rate decreases with a decrease in the calculated flow rate, although with lower flow rates, the permissible circumferential velocity is greater. Due to the lower values of $\lambda_{u h_{c r i t}}$, the maximum performance of the stages with $\Omega=1.0$ is less, other factors being equal. 

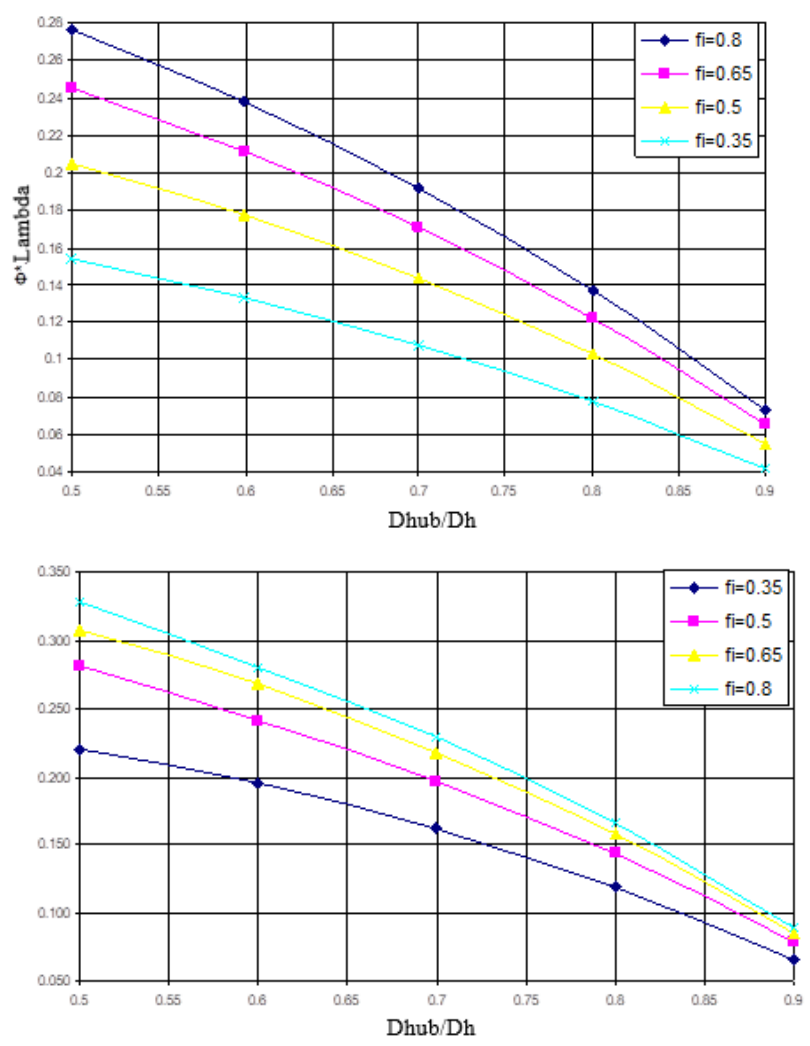

Fig. 5a, 5b. Influence of the hub-tip ratio and the calculated flow coefficient on the maximum dimensionless flow rate $\bar{\Phi}_{\text {lim }}=\Phi \cdot \lambda_{u h_{\text {crit }}}$.

At the top $\Omega=1.0$, at the bottom $\Omega=0.5$.

The studied factors have a significant impact on the efficiency of the stages - Fig. 6a, 6b. When analyzing the results, we pay attention to the low level of efficiency calculated according to the "classical" recommendations of Western authors, presented, for example, in [22]. However, it is also indicated that the efficiency of carefully developed axial stages lies in the range of $0.86-0.92$, while domestic sources indicate the efficiency of model stages exceeding $95 \%$ at $\Omega=0.5$ and $93 \%$ at $\Omega=1.0$ [26].

We also note that the efficiency of stages with reactivity of 1.0 increases with the increase of the calculated flow coefficient (in the studied range). At $\phi_{\mathrm{p}}<0.65$, the drop in efficiency becomes very strong, and at $\phi_{\mathrm{p}}=0.35$, calculations predict an almost unacceptable efficiency for axial stages.

For stages with reactivity of 0.5 , the maximum efficiency corresponds to flow coefficient of 0.5 , at which the kinetic energy and Euler head are in the optimal ratio. With flow rate of 0.65 , the achievable efficiency is slightly lower.
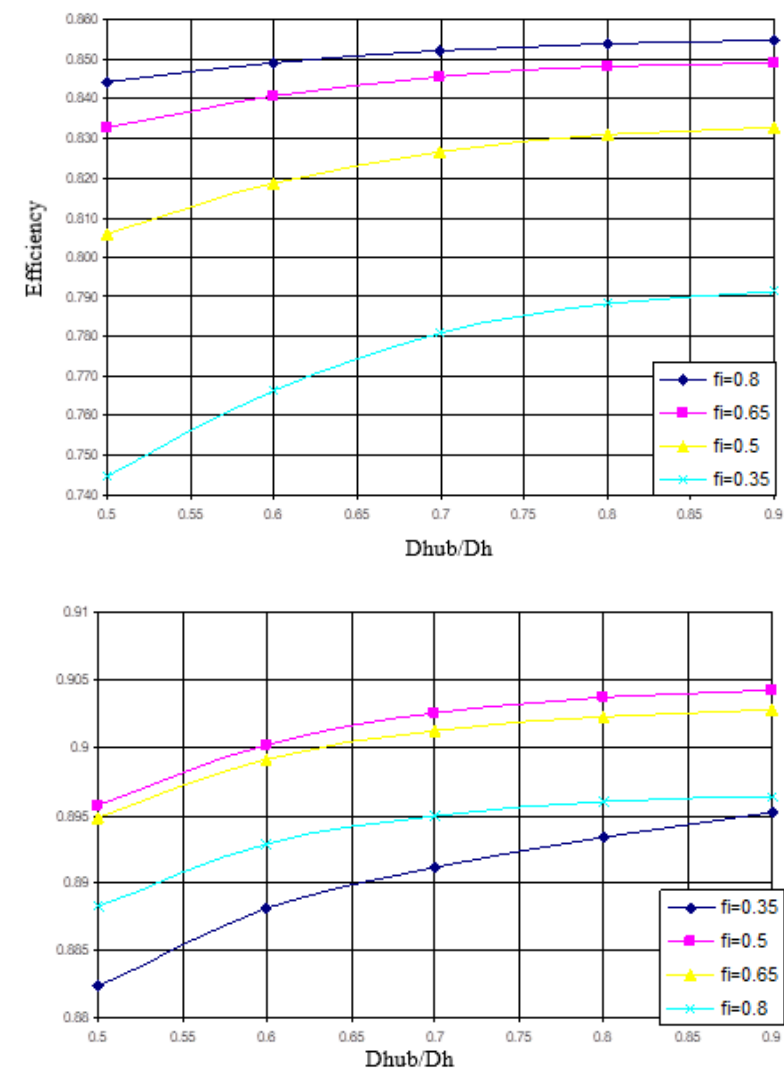

Fig. $6 a, 6 b$. Influence of the hub-tip ratio and the calculated flow coefficient on the stages efficiency.

At the top $\Omega=1.0$, at the bottom $\Omega=0.5$.

\subsection{Comparison of the characteristics of reactivity} levels with 0.5 and 1.0

To balance the empirical coefficients $\mathrm{Xi}$ in equations (13) - (15), the characteristics of the model stages K50-1 and K-100-1 from the monograph of A.P. Goflin [26] were selected. The calculations were based on published data on the stages, summarized in the table below. When processing the results, the following was accepted:

- construction of the spatial flow according to the law $\mathrm{c}_{\mathrm{ur}}=$ const,

- average calculated radius was defined as the root-mean-square,

- the values of the loading factor and efficiency coefficient calculated in [26] from the adiabatic head were assumed to be equal to the polytropic ones - due to the insignificant ratio of the stages pressures, even at maximum circumferential velocities,

- diffusion airfoil factor of the $\mathrm{Im}$ in the calculations was determined by selecting the loading factor equal to the measured one.

Based on the above calculation, the parameters were determined, summarized in the following table. 
Table 1. Parameters of model stages from [26] used in the design analysis of characteristics

\begin{tabular}{|c|c|c|c|c|c|c|c|c|}
\hline $\begin{array}{c}\text { para } \\
\text { meter } \\
\backslash \\
\text { stage }\end{array}$ & $\begin{array}{l}\text { Dhu } \\
\text { b/Dh }\end{array}$ & $\begin{array}{l}1 / \\
\mathrm{B}\end{array}$ & $\begin{array}{l}\text { (th/ } \\
\text { B) } \\
\text { Im }\end{array}$ & $\begin{array}{l}\text { (th/B } \\
\text { )GD }\end{array}$ & $\begin{array}{c}\lambda_{u h} \\
\mathrm{ma} \\
\mathrm{x} / \\
\mathrm{mi} \\
\mathrm{n}\end{array}$ & $\phi_{h}$ & $2 \psi_{\text {ad } h}$ & $\eta_{a d}$ \\
\hline $\begin{array}{c}\mathrm{K}- \\
50-1\end{array}$ & $\begin{array}{c}0.60 \\
0\end{array}$ & $\begin{array}{l}1 . \\
63\end{array}$ & $\begin{array}{c}1.1 \\
9\end{array}$ & 0.77 & $\begin{array}{c}0.6 \\
36 / \\
0.8 \\
91 \\
\end{array}$ & $\begin{array}{c}0.480 \\
\left(\lambda_{u h}=0\right. \\
.636)\end{array}$ & $\begin{array}{c}0.480 \\
\left(\lambda_{\text {uh }}=0\right. \\
.636)\end{array}$ & $\begin{array}{c}0.955 \\
\left(\lambda_{u h}=0\right. \\
.636)\end{array}$ \\
\hline $\begin{array}{c}\text { K- } \\
100-1\end{array}$ & $\begin{array}{c}0.50 \\
5\end{array}$ & $\begin{array}{l}2 . \\
04\end{array}$ & $\begin{array}{c}1.0 \\
9\end{array}$ & 1.31 & $\begin{array}{l}0.5 \\
09 / \\
0.8 \\
53 \\
\end{array}$ & $\begin{array}{c}0.420 \\
\left(\lambda_{h h}=0\right. \\
.509)\end{array}$ & $\begin{array}{l}0.420 \\
\left(\lambda_{u h}=0\right. \\
.509)\end{array}$ & $\begin{array}{l}0.930 \\
\left(\lambda_{u h}=0\right. \\
.509)\end{array}$ \\
\hline
\end{tabular}

Table 2. Parameters of model stages from [26] used in the design analysis of characteristics Parameters of model stages from [26], recalculated for design analysis of characteristics

\begin{tabular}{|c|c|c|c|c|}
\hline $\begin{array}{c}\text { parameter } \\
\text { Itage }\end{array}$ & $\bar{r}_{h}$ & $\begin{array}{c}\phi_{\text {des }} \\
=\phi_{h} \\
* \bar{r}_{h}\end{array}$ & $\begin{array}{l}\psi_{\mathrm{Th}} \\
=2 \psi_{\mathrm{a} d h} \\
/ 2 \eta_{\mathrm{a} d}\end{array}$ & $F_{D \text { Im }}$ \\
\hline $\mathrm{K}-50-1$ & 1.213 & 0.582 & 0.251 & 0.465 \\
\hline $\mathrm{K}-100-1$ & 1.262 & 0.530 & 0.242 & 0.413 \\
\hline
\end{tabular}

Figure 7 shows the calculated characteristics of a number of stages with reactivity of 1.0 with the design parameters from the tables above. The empirical coefficients $\mathrm{Xi}$ were selected in such a way that the efficiency characteristics at different velocity coefficients approximately correspond to the data for the K-100-1 stage from [26].

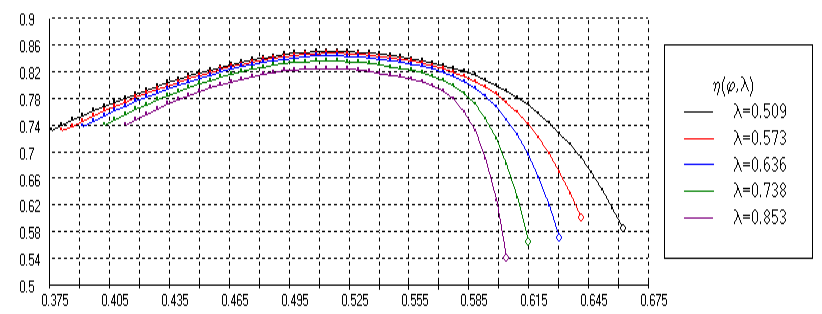

Fig. 7a. Calculated characteristics of the efficiency of stages with reactivity of 1.0 at different $\lambda_{u h}$

The calculation was performed at values $\lambda_{u h}$, corresponding to the circumferential velocities at which the K-100-1 stage was tested. Figures 8 and 9 show the calculated characteristics of the polytropic pressure coefficient at the outer radius, and the pressure ratio.

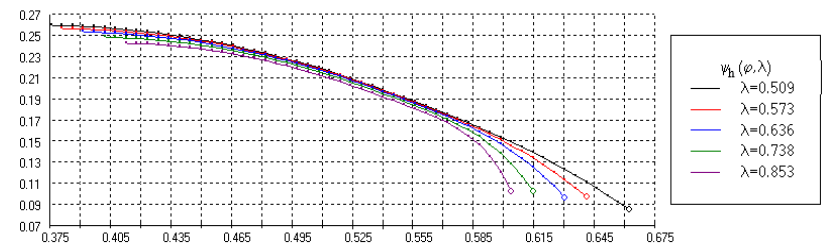

Fig. 7b. Calculated characteristics of the stages loading factor with reactivity of 1.0 at different $\lambda_{u h}$

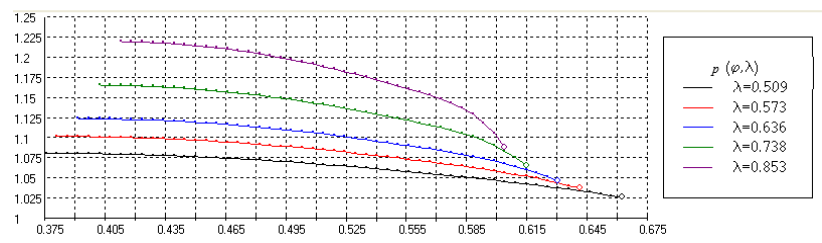

Fig. 7c. Calculated characteristics of the stages pressure ratio with reactivity of 1.0 at different $\lambda_{u h}$

As in the analysis of limit parameter stages, attention is drawn to the low calculated efficiency of stages with a reactivity of 1.0 - less than $86 \%$ at the lowest rotation velocity - against $93 \%$ according to data [26] for a similar stage. It should also be noted that even at $\lambda_{u h}=0.738$, the local flow velocity at the shroud of the impeller exceeds the sound velocity, namely, $\lambda_{w \max }=1.011$. At the maximum circumferential velocity at which the K-100-1 stage was tested, corresponding to $\lambda_{u h}=0.853$, the local flow velocity at the shroud of the impeller is already noticeably higher than sound velocity. In this case, $\lambda_{\text {wmax }}=1,15$.

In accordance with the applied principle of characteristics modeling, the empirical coefficients $\mathrm{Xi}$ should be universal, suitable for any stages modeling, since the empirical basis of calculation is the universal characteristics of flat grids. Universal characteristics generalize the results of purge of a large number of grids in wind tunnels of various shapes.

The characteristics of the stages with reactivity of 0.5 were calculated from the values of $\mathrm{Xi}$, which approximately correspond to the characteristics of the K-100-1 stage. Figure 8 shows the calculated characteristics of a number of stages with reactivity of 0.5 with the design parameters from the above tables - that is, corresponding to the K-50-1 stage from [26].

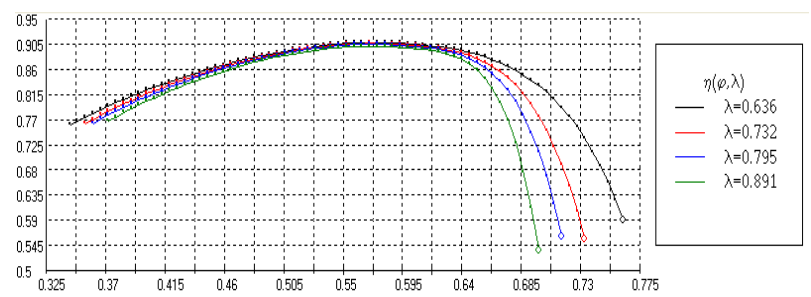

Fig. 8a. Calculated characteristics of the efficiency of stages with reactivity of 0,5 at different $\lambda_{u h}$ 


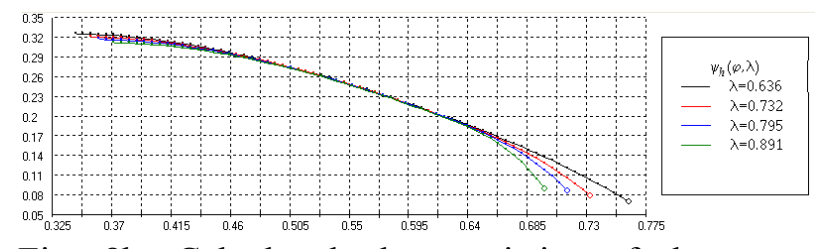

Fig. 8b. Calculated characteristics of the stages loading factor with reactivity of 0,5 at different $\lambda_{u h}$

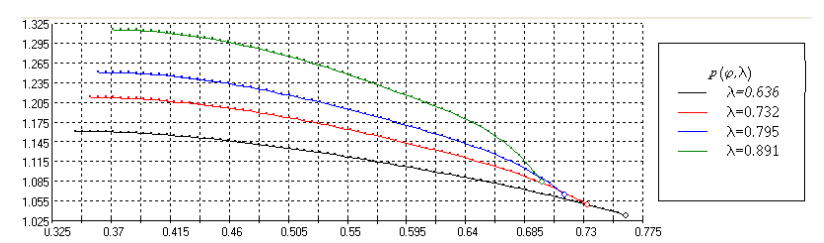

Fig. 8c. Calculated characteristics of the stages pressure ratio with reactivity of 0,5 at different $\lambda_{u h}$

When considering the results, we pay attention to the fact that the efficiency of stages with $\Omega=0.5$ is $4 \%$ higher than that of stages with reactivity of 1.0 , but significantly lower than the data given in [26]. Local values of the velocity coefficient do not reach one at all $\lambda_{u h}$. Accordingly, the efficiency values in the calculated flow modes do not differ much in the studied range $\lambda_{u h}$.

Attention is drawn to the obvious discrepancy between the width of the working zone of the stages with $\Omega=0.5$ according to the calculation and according to the test data from [26] - the calculated characteristics have an improbably large "surge margin" - ratio $\phi o p t_{\text {min }}$. If, according to the test data, this ratio is 0.82 at $\lambda_{u h}=0.636(\mathrm{uh}=200 \mathrm{~m} / \mathrm{s})$, then this ratio is calculated to be 0.60 , which is completely improbable for an axial stage.

\section{Conclusion}

This article completes the publication of the results of the calculated analysis of axial stages, based on the use of the results of flat grids purge - see also [1], [2].

The authors suppose that the advantage of the ODOS-GP04 calculation program can be considered a fairly high information content in the analysis of the influence of design parameters, velocity and flexibility (that is, the possibility of qualitative and quantitative analysis of all the main gas-dynamic solutions in the design of the flow path). The program is intended primarily for illustrating a lecture course on gas dynamics of axial compressors and course design.

Further research in this direction will be associated with the modernization of mathematical models and the expansion of the capabilities of computer programs based on them. For calculation trans- and supersonic compressors, it is necessary to take into account the occurrence of direct and oblique shock waves, possible separation of the flow and the fact that during supersonic flow there is no flow restructuring at the inlet to the blade cascade.

The attention of specialists may be attracted by a noticeable discrepancy between some of the calculation results with the established ideas about the efficiency and characteristics of axial stages. First, we are talking about a noticeably lower efficiency of the stages calculated according to the generally accepted empirical formulas from [22], [23]. Second, the Russian literature usually indicates a wider area of operation of stages with reactivity of 1.0. The above calculation data can be interpreted as a refutation of this opinion.

\section{Acknowledgment}

This work was financially supported by the Ministry of Science and Higher Education of the Russian Federation in the framework of the Federal Program "Research and Development in Priority Directions for the Development of the Scientific and Technological Complex of Russia for 2014-2020". Agreement on the provision of a grant in the form of subsidy No. 075-15-2019-1710 dated December 2, 2019 (internal number 05.608.21.0275). Unique identifier: RFMEFI60819X0275.

\section{References:}

[1] Yu.B. Galerkin, Yu.A. Popov, A.Yu. Prokofyev, Analysis of the efficiency of axial compressors elementary grids based on the flat grids blowdown data. Compressor equipment and Pneumatics, 2005, No. 1, pp. 13-20.

[2] Yu.B. Galerkin, Yu.A. Popov, Analysis of the efficiency of spacial blade compressors grids based on the flat grids blowdown data, Compressor equipment and Pneumatics, 2005, No. 3, pp. 33-38.

[3] Yu.S. Podobuev, K.P. Seleznev, Theory and calculation of axial and centrifugal compressors, M., L.: Mashgiz, 1957, pp. 320

[4] K.V. Kholshevnikov, Theory and calculation of aviation blade machines. Moscow: Mechanical Engineering, 1986, pp. 432

[5] I.V. Brusilovsky, Aerodynamic calculation of axial fans. Moscow: Mechanical Engineering, 1986, pp. 284

[6] Method of aerodynamic calculation of the flow part of an axial compressor for stationary installations. RTM 24.020.17-73. M.: MTTЭM, 1975, pp. 204

[7] V.V. Semov, Experimental study of a type K-3 compressor stage (K-50-3) with a small blades elongation. EMS, No. 10, 1968, pp. 30-34. 
[8] M. Banjac, M.V. Petrovic, Development of method and computer program for multistage axial compressor design: part I - mean line design and example cases, Proceedings of ASME Turbo Expo 2018 Turbomachinery Technical Conference and Exposition GT2018 June 11-15, 2018, Oslo, Norway

[9] M. Banjac, M.V. Petrovic, Development of method and computer program for multistage axial compressor design: part II twodimensional design and validation using CFD. Proceedings of ASME Turbo Expo 2018 Turbomachinery Technical Conference and Exposition GT2018 June 11-15, 2018, Oslo, Norway

[10] Olivier Adam, Olivier Léonard. A quasi-one dimensional model for axial compressors

[11] Chen Yang, $\mathrm{Hu} \mathrm{Wu}$, Jinguang Yang and Michele Ferlauto, Time-marching throughflow analysis of multistage axial compressors based on a novel inviscid blade force model, Journal of aerospace engineering, 2019, DOI:10.1177/0954410019840588

[12] Chen Yang, Hu Wu, Yan Liang, A Novel ThreeDimensional Inverse Method for Axial Compressor Blade Surface Design, Arabian Journal for Science and Engineering

[13] Ioannis Kolias, Alexios Alexiou, Nikolaos Aretakis, Konstantinos Mathioudakis. Direct integration of axial turbomachinery preliminary aerodynamic design calculations in engine performance component models. Proceedings of ASME Turbo Expo 2018 Turbomachinery Technical Conference and Exposition GT2018 June 11-15, 2018, Oslo, Norway

[14] T. Li, Axial compressor performance prediction using improved streamline curvature approach, T. Li, Y. Wu, H. Ouyang, X. Qiang, Proceedings of ASME Turbo Expo 2018 Turbomachinery Technical Conference and Exposition GT2018 June 11-15, 2018, Oslo, Norway

[15] Youwei He, Jinju Sun, Peng Song, Xuesong Wang, Da Xu. Development of a multi-objective preliminary design optimization approach for axial flow compressors, Proceedings of ASME Turbo Expo 2018 Turbomachinery Technical Conference and Exposition GT2018 June 11-15, 2018, Oslo, Norway

[16] John Kidikian, Marcelo Reggio, Off-design prediction of transonic axial compressors, part 1: mean-line code and tuning factors. Proceedings of ASME Turbo Expo 2018. June 11-15, 2018, Oslo, Norway
[17] John Kidikian, Marcelo Reggio, Off-design prediction of transonic axial compressors, part 2: generalized mean-line loss modelling methodology, Proceedings of ASME Turbo Expo 2018 June 11-15, 2018, Oslo, Norway

[18] Xuesong Wang; Jinju Sun; Peng Song; Youwei $\mathrm{He}$; Da Xu Three-dimensional blade shape optimization for a transonic axial flow compressor through incorporating surrogate model and sequental sampling, Proceedings of ASME Turbo Expo 2018 Turbomachinery Technical Conference and Exposition GT2018 June 11-15, 2018, Oslo, Norway

[19] Sangjo Kima, Kuisoon Kima, Changmin Sonb. Three-dimensional unsteady simulation of a multistage axial compressor with labyrinth seals and its effects on overall performance and flow characteristics, Aerospace Science and Technology, 86, (2019), pp. 11-693

[20] Yuri Galerkin, Yuri Popov, Optimal primary design of industrial axial compressor flow path, International Conference on Compressors and their Systems. London. City University, UK, 2009, pp. 319-329.

[21] S. Lieblein, Experimental flow in 2D cascades, 1956. Chapter V1 of The Aerodynamic Design of Axial Flow Compressor. Reprinted NASA SP 36 in 1965.

[22] N.A. Cumpsty, Compressor Aerodynamics, Longman Scientific and Technical, 1989, pp. 509

[23] A.R. Howell, Fluid dynamics of axial compressors, Proc. I Mech. E. 153: 441, 82, 1945

[24] K.P. Seleznev, Yu.b. Galerkin, etc. Theory and calculation of turbine superchargers, Mechanic engineering, 1986.

[25] Proceedings of the Scientific School of Compressor Engineering of SPbSTU. Under the editorship of prof. Yu.B. Galerkin. Saint Petersburg, 2000. pp. 443, with Fig.

[26] A.P. Goflin, Aerodynamic calculation of the flow path of axial compressors for stationary installations, Mashgiz, 1959.

\section{Contribution of individual authors to the creation of a scientific article (ghostwriting policy)}

A.I. Borovkov, Yu.B. Galerkin, V.A. Chernikov have organized the researches

Yu.A. Popov, O.A. Solovieva, A.A. Drozdov were developed 2D mathematical model 
A.F. Rekstin, L.N. Marenina were provide numerical resarches

Follow: www.wseas.org/multimedia/contributorrole-instruction.pdf

This work was financially supported by the Ministry of Science and Higher Education of the Russian Federation in the framework of the Federal Program "Research and Development in Priority Directions for the Development of the Scientific and Technological Complex of Russia for 2014-2020". Agreement on the provision of a grant in the form of subsidy No. 075-15-2019-1710 dated December 2, 2019 (internal number 05.608.21.0275). Unique identifier: RFMEFI60819X0275.

\section{Creative Commons Attribution License 4.0 (Attribution 4.0 International, CC BY 4.0)}

This article is published under the terms of the Creative Commons Attribution License 4.0

https://creativecommons.org/licenses/by/4.0/deed.en_US 